\title{
Reducing FEC-Complexity in Cross-Layer Predictable Data Communication
}

\author{
Pablo Gil Pereira and Thorsten Herfet \\ Telecommunications Lab, \\ Saarland Informatics Campus, D-66123 Saarbrücken, Germany \\ Email: \{gilpereira, herfet\}@cs.uni-saarland.de
}

\begin{abstract}
The PRRT protocol enables applications with strict performance requirements such as Cyber-Physical Systems, as it provides predictably low, end-to-end delay via cross-layer pacing and timely error correction via Hybrid ARQ (HARQ). However, the implemented HARQ uses computationally complex Maximum Distance Separable (MDS) codes to generate redundancy. In this paper we propose code partitioning for the complexity reduction of MDS codes, thereby enabling their deployment on constrained embedded devices.
\end{abstract}

Index Terms-Forwad error coding, transport protocols, crosslayer optimization

\section{INTRODUCTION}

Cyber-Physical Systems (CPS) integrate physical and digital processes via control loops, often using communication networks in distributed deployments. In order to guarantee a successful operation, CPS require predictable reliability and delay, which are difficult to achieve due to the inherent design of computation and communication systems [1]. The Predictably Reliable Real-time Transport (PRRT) [2] protocol can optimize its configuration to meet the delay constraints of a system with cross-layer pacing [3], which keeps the system and network buffers empty to minimize the end-to-end delay. Timely reliability is achieved with a Hybrid ARQ (HARQ) scheme that extends the error correction in lower layers to cope with losses not bound to the link within a target delay.

Since the transport layer works at the packet level, the block lengths are usually several orders of magnitude shorter than those at the physical layer, especially when operating under delay constraints. Therefore, Minimum Distance Separable (MDS) codes have been used [4]-[6] because they are more suitable for short codes than LDPC or polar codes as they do not require excess packets. However, they have higher complexity, which may result in large en- and decoding delays when deployed on devices with limited computation capabilities. This paper analyses the impact of the complexity of MDS codes on the design of transport protocols providing predictable reliability and delay, using PRRT as a proof of concept. Additionally, we propose code partitioning to efficiently reduce the complexity of MDS codes by $50 \%$ with only a slight increase in the redundancy information.

The work is supported by the German Research Foundation (DFG) within SPP 1914 "Cyber-Physical Networking" under grant 315036956

\section{Code Partitioning}

While the Automatic Repeat reQuest (ARQ) delay is mainly dominated by the round-trip time, the inter-packet time dominates the Forward Error Coding (FEC) delay. As a result, the information-theoretical optimum under delay constraints will be a combination of both, which HARQ implements [2]. Although FEC encoding and decoding delays are usually negligible when the protocol runs on powerful machines, this does not hold true when it runs on more constrained devices. Both the encoding and decoding of MDS codes entail a matrixvector multiplication, whereas the decoding process requires an additional matrix inversion. If systematic codes are used, MDS codes achieve encoding complexity $\mathcal{O}(k p)$ and decoding complexity $\mathcal{O}\left(k p^{2}\right)$ [4], with $k$ the block length and $p$ the number of parity packets.

The algorithm to find the optimal $p$ and $k$ is outside of the scope of this paper and the reader is referred to [2] for more details about it. However, it is relevant to know that this algorithm finds the largest $k$ that meets the target delay $\left(D_{T}\right)$ and packet loss rate $\left(P L R_{T}\right)$ constraints because larger codes result in lower RI than shorter ones. This property is depicted in Table I] where $n=k+p$ has been calculated such that $P L R_{F E C} \leq P L R_{T} . P L R_{F E C}$ is the FEC packet loss rate as given by Eq. 1. for which we have assumed a Binary Erasure Channel (BEC) with erasure probability $p_{e}$ and have defined the random variable $I_{k}^{n}$, which represents the number of packet losses in a code $C(n, k)$. Although the PLR analysis is also available for channels with memory (i.e., Gilbert-Elliott model), this does not change the basic findings and hence it is omitted for clarity in this paper. Since $k$ has a major impact on the code complexity, we propose code partitioning to split the block into two independent blocks instead of using the largest $k$. As a result, the used $k$ and $p$ are halved but the encoding and decoding operations run twice for the original block length, which nevertheless reduces the complexity to $\mathcal{O}\left(\frac{k p}{2}\right)$ for encoding and $\mathcal{O}\left(\frac{k p^{2}}{4}\right)$ for decoding. The packet loss rate of partitioned codes $\left(P L R_{F E C}^{p a r t}\right)$ is given in Eq. 4, where $J_{k_{1}, k_{2}}$ is a random variable representing the packet losses in a partitioned code and $k_{\max }=\left\lceil\frac{k}{2}\right\rceil$.

$$
P L R_{F E C}=\frac{1}{k} E\left[I_{k}^{n}\right]=\frac{1}{k} \sum_{i=1}^{k} i \cdot P\left(I_{k}^{n}=i\right)
$$




\begin{tabular}{|c|c|c|c|c|c|c|}
\hline$p_{e}$ & 0.01 & 0.03 & 0.05 & 0.07 & 0.09 & 0.1 \\
\hline$n(k=40)$ & 44 & 48 & 50 & 53 & 55 & 56 \\
\hline$n(k=80)$ & 86 & 91 & 95 & 98 & 102 & 104 \\
\hline
\end{tabular}

Table I

OPTIMUM MDS CODE CONFIGURATION TO MEET $P L R_{T}=10^{-5} \mathrm{FOR}$ DIFFERENT BLOCK LENGTHS

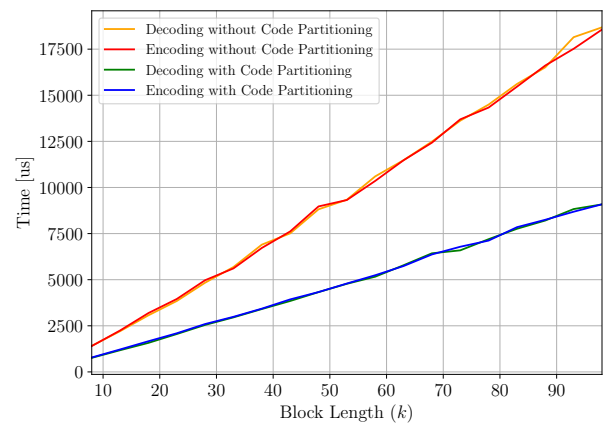

Figure 1. PRRT's MDS code encoding and decoding times with and without code partitioning for $m=8$ redundancy packets.

$$
\begin{aligned}
& P\left(I_{k}^{n}=i\right)= \begin{cases}\sum_{e=0}^{n-k}\left(\begin{array}{l}
n \\
e
\end{array}\right) p_{e}^{e}\left(1-p_{e}\right)^{n-e} & i=0 \\
\sum_{e=\max (n-k+1, i)}^{n-k+i} p\left(\begin{array}{l}
n \\
e
\end{array}\right) \frac{\left(\begin{array}{l}
k \\
i
\end{array}\right)\left(\begin{array}{l}
n-k \\
e-i
\end{array}\right)}{\left(\begin{array}{l}
n \\
e
\end{array}\right)} & 1 \leq i \leq k\end{cases} \\
& p\left(\begin{array}{l}
n \\
e
\end{array}\right)=\left(\begin{array}{l}
n \\
e
\end{array}\right) p_{e}^{e}\left(1-p_{e}\right)^{n-e} \\
& P L R_{F E C}^{\text {part }}=\frac{1}{k} E\left[J_{k_{1}, k_{2}}\right]=\frac{1}{k} \sum_{j=1}^{k} j \cdot P\left(J_{k_{1}, k_{2}}=j\right) \\
& P\left(J_{k_{1}, k_{2}}=j\right)=\sum_{e=\max \left(0, j-k_{\max }\right)}^{\min \left(k_{\max }, j\right)} P\left(I_{k_{1}}^{n_{1}}=e\right) \cdot P\left(I_{k_{2}}^{n_{2}}=j-e\right)
\end{aligned}
$$

The FEC delay is impacted in two ways: i) the decrease in encoding and decoding delays and ii) the increase in $p$ when $P L R_{F E C}^{\text {part }}>P L R_{T} \geq P L R_{F E C}$.

\section{EvAluATION}

PRRT's HARQ implementation using code partitioning has been executed on a Raspberry Pi Zero W running Raspbian Buster with Linux kernel 4.19, which has been configured to run as few processes as possible to reduce the interference with the experiment. Figure 1 shows the measurements of the encoding and decoding delays. As predicted in Section III, the encoding delay is halved when code partitioning is employed. A surprising result is that the encoding and decoding delays are the same, even though the decoding needs to perform a matrix inversion. The matrix inversion code has been isolated, resulting in $0.25 \mathrm{~ms}$ delay for $k=100$, which shows that the decoding delay is dominated by the matrix-vector multiplication of $1500 \mathrm{~B}$ packets and the inversion is negligible.
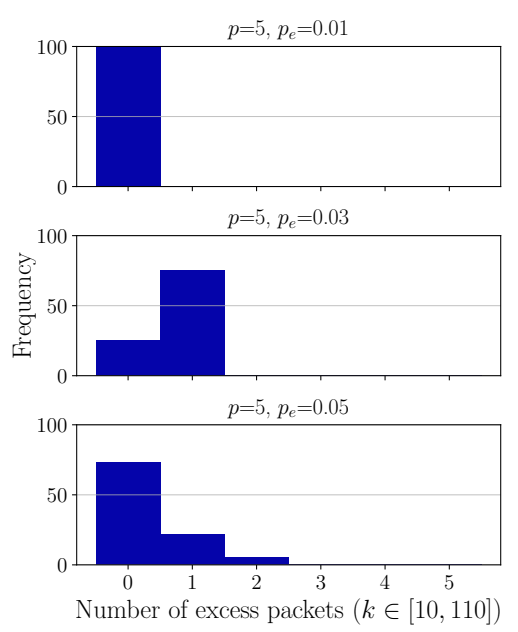

Figure 2. Required number of excess packets in code partitioning for 5 redundancy packets $(p=5)$ and erasure probability $p_{e}=\{0.01,0.05,0.1\}$, so that $P L R_{F E C}-P L R_{F E C}^{\text {part }} \leq 0.001$.

The analysis of the required excess packets is depicted in Figure 2, which shows three histograms for a fixed number of parity packets $(p=5)$ and $p_{e}=\{0.01,0.05,0.1\}$. The three configurations consider $k \in[10,110]$. In this case, excess packets have alternately been distributed between both codes until $P L R_{F E C}-P L R_{F E C}^{\text {part }} \leq \delta$, with $\delta=0.001$. As depicted in the picture, in most cases code partitioning is not penalized by an increase in RI.

\section{CONCLUSION}

PRRT is an example of a transport protocol using HARQ to provide predictable reliability and delay, applying an MDS code to generate redundancy to recover from losses. However, packet encoding and decoding have high computational complexity, making them unsuitable for energy and computationally constrained devices. In this paper, we analyze the complexity of MDS codes and propose code partitioning, an approach to reduce the said complexity by $50 \%$ with only a slight increase in the required redundancy information.

\section{REFERENCES}

[1] E. A. Lee, "Cyber Physical Systems: Design Challenges," in Proceedings of the 11th IEEE International Symposium on Object and ComponentOriented Real-Time Distributed Computing (ISORC), pp. 363-369, IEEE, 2008.

[2] M. Gorius, Adaptive Delay-constrained Internet Media Transport. PhD thesis, Saarland University, 2012.

[3] A. Schmidt, S. Reif, P. G. Pereira, T. Hönig, T. Herfet, and W. SchröderPreikschat, "Cross-Layer Pacing for Predictably Low Latency," Proceedings of the 6th International Workshop on Ultra-Low Latency in Wireless Networks (ULLWN), 2019.

[4] L. Rizzo, "Effective erasure codes for reliable computer communication protocols," ACM SIGCOMM Computer Communication Review, vol. 27, no. 2, pp. 24-36, 1997.

[5] J. Lacan and J. Fimes, "Systematic MDS Erasure Codes Based on Vandermonde Matrices," IEEE Communications Letters, vol. 8, no. 9, pp. 570-572, 2004.

[6] A. Soro and J. Lacan, "FNT-based Reed-Solomon Erasure Codes," in 2010 7th IEEE Consumer Communications and Networking Conference, pp. 1-5, IEEE, 2010. 